\title{
REALIDADE VIVENCIADA PELOS ENFERMEIROS EM PROGRAMA SAÚDE DA FAMÍLIA
}

\author{
EXPERIENCED REALITY BY NURSES IN FAMILY HEALTH PROGRAM CONTEX
}

LA REALIDAD VIVIDA POR LOS ENFERMEROS EN EL PROGRAMA SALUD DE LA FAMILIA

\author{
Maria de Lourdes Centa* \\ Leda Maria Alburquerque ${ }^{* *}$ \\ Marcos Augusto Moraes Arcoverde ${ }^{\star \star *}$
}

* Doutora em Enfermagem, professora do Programa de Pós-Graduação em Enfermagem da UFPR. Coordenadora do Grupo de Estudos Saúde Família e Desenvolvimento.

** Enfermeira da Secretaria Municipal de Curitiba. Mestranda do Programa de Pós-Graduação em Enfermagem da UFPR.

*** Mestrando do Programa de Pós-Graduação em Enfermagem da UFPR.

RESUMO: Dentro das Políticas de Saúde preconizadas pelo governo brasileiro, o Programa de Saúde da Família (PSF) vem sendo priorizado para assistir/cuidar da saúde das famílias. Sua principal finalidade é reorganizar a prática de atenção à saúde, substituindo o modelo tradicional, procurando levar a saúde para mais perto das famílias e, com isso, melhorar a qualidade de vida da população. O PSF baseia-se em ações inter e multidisciplinar, onde os profissionais procuram atender as necessidades básicas da família através de ações eficientes, de alta resolutividade e baixo custo, procurando proporcionar-lhes melhores qualidade de vida. Neste processo o enfermeiro interage com as famílias, assistindo e cuidando de seus membros. Baseado nas ações desenvolvidas por estes profissionais foi que nos propusermos a desenvolver este estudo cujo objetivo é retratar a experiência vivida pelos enfermeiros que atuam em PSF. Utilizou-se a metodologia qualitativa, estudo exploratório descritivo. Os dados foram obtidos através de um questionário semi-estruturado. Seguiram-se as normas da resolução 169/96 que dispõem sobre pesquisa com seres humanos. Os dados foram discutidos, analisados e categorizados de forma a retratar o pensar e o agir dos enfermeiros, os quais nos permitiram construir as seguintes categorias: ATENDIMENTO DIFERENCIADO; DIFICULDADES ENCONTRADAS; SENTIMENTOS EXPERIENCIADOS, o que nos levou a categoria central: REALIDADE VIVENCIADA PELOS ENFERMEIROS EM PSF. Conclui-se que os enfermeiros que atuam em PSF necessitam rever suas habilidades, conhecimentos e formas de atuação para conseguir desenvolver ações de saúde eficientes, que realmente proporcionem resolutividade aos problemas apresentados pelas famílias e com isso melhorem sua qualidade de vida.

PALAVRAS-CHAVE: Enfermeiros; Programa Saúde da Família; Prática profissional.

ABSTRACT: Among Brazilian's government politics, Family Health Program - FHP has been prioritized to take care of families. Its main purpose is reorganize the health system and substitute the traditional model, taking the health care more close to the families and, with this, improves life quality of population. The FHP is based on Interdisciplinary action, where the professionals take care of basic family's necessities through efficient actions, high resolvability and low cost, providing better life quality to them. In this process the nurse interacts with families attending and taking care of its members. Based on those professionals' actions, the objective of this study is to show the nurses experience acting on FHP. It was used qualitative methodology, descriptive exploratory study. Semi-structured questionnaire was used to collect the information. The terms of 169/96 resolution was followed. The questions were argued, analyzed and categorized in order to portray the nurses thinking and acting in this program. They allowed to construct the following categories: DIFFERENTIATED ATTENDANCE; JOINED DIFFICULTIES; FEELINGS EXPERIENCIED, which gave us the central category: EXPERIENCED REALITY FOR NURSES IN PSF. The conclusion tells that the nurses need to review its abilities, knowledge and performance to obtain efficient actions on health that will really provide resolvability to the problems presented by families and with this improve its life quality.

KEY WORDS: Nurses; Family Health Program; Professional practice.

RESUMEN: Entre las políticas de la salud predicadas por el gobierno brasileño, el PSF esta siendo electo socorrer/cuidar de la salud de las familias. Su propósito principal es reorganizar la práctica de atención a la salud, substituyendo el modelo tradicional, llevando así la salud para mas cerca de las familias. El PSF tiene como base acciones de inter y multidisciplinas, donde los profesionales buscan atender las necesidades básicas de la familia con acciones eficientes, solucionables y de bajo costo, cuya finalidad es la proporcionarles una mejor calidad de vida. En este proceso el Enfermero participa socorriendo y cuidando de los miembros de la familia, con base en las acciones desarrolladas por estes profissionales propusimos realizar este estudio, con el objetivo de retratar la experiencia vivida por ellos en el PSF. Fue usado el método cualitativo, explorativo - discriptivo, siendo respetada la resolución 169/96 que trata de pesquisa com seres humanos. Los datos fueram cogidos a través de un cuestionario semi-estructurado e posteriormente discutidos, analizados y separados de forma a retratar el pensamiento y actuacion de los enfermeros que trabajan en este programa. Los dados nos han permitido construir las categorias que seguen: ATENDIMENTO DIFERENCIADO; DIFICULTADES ENCONTRADAS; EXPERIENCIAS VIVIDAS, lo que nos llevó a la categoría central: LA REALIDAD VIVIDA POR LOS ENFERMEROS EN EL PSF. Concluimos que los enfermeros que actúan en el PSF necesitan estudiar sus habilidades, conocimientos y maneras de actuar para que puedan desarrollar acciones eficientes de salud, que realmente proporcionen solución a los problemas presentados por las familias y consecuentemente mejoren su calidad de vida.

PALABRAS-CLAVE: Enfermeros; Programa de Salud de la Família; Práctica profesional.

Recebido em: 11/08/2003

Aceito em: $15 / 12 / 2003$
Maria de Lourdes Centa

Rua Pará, 1235 - Água Verde

80610-020 - Curitiba - PR 


\section{INTRODUÇÃO}

O período pós 1995 foi marcado pela expansão da Atenção Básica de Saúde, a qual está reorientando a reorganização lógica assistencial do SUS. ${ }^{1}$ Percebe-se que o Programa Saúde da Família (PSF) é parte dessa expansão, pois a idéia é garantir que os programas sociais atinjam os seguimentos mais vulneráveis da sociedade. ${ }^{2}$

Como modelo estratégico para a saúde, podemos considerar o PSF como uma nova forma de reestruturação, um novo modelo do processo de trabalho na saúde, o que ajudaria cumprir a Lei 8080/ 90 do Ministério da Saúde (MS), que trata a saúde como um direito fundamental do cidadão brasileiro e dever do Estado. ${ }^{3}$

Em 1997, o MS institui o PSF como uma estratégia para a reorientação do modelo assistencial, quando então, assume o desafio de colocar em seu plano de Ações e Metas Prioritárias as estratégias do PSF e de Agentes Comunitários de Saúde (ACS), como caminhos possíveis da reorganização da atenção básica em saúde. ${ }^{4}$

Segundo esse órgão, o PSF não é uma estratégia desenvolvida para a atenção exclusiva a grupos específicos da população, mas sim trabalha com o princípio da vigilância à saúde, apresentando como uma de suas características a atuação inter e multidisciplinar. $^{4}$

Os profissionais que atuam neste programa devem prestar assistência integral e contínua, com resolutividade e boa qualidade à população de sua área de abrangência, atendendo as famílias inseridas em seu contexto social, de forma humanitária, eficaz e com resolutividade. Para isso eles devem estabelecer vínculo com a população, contribuir para a democratização do conhecimento do processo saúde-doença, fazendo com que a saúde seja reconhecida como um direito de cidadania e, portanto, expressão da qualidade de vida. Estes profissionais, também, devem estimular a organização da comunidade para que ela torne-se co-responsável pelas ações desenvolvidas pelo programa. $^{5}$

Um excelente avanço do PSF foi unir e formar equipe multiprofissionais. O MS preconiza que uma equipe de PSF deve ser constituída de no mínimo um médico, um enfermeiro, um auxiliar de enfermagem e de quatro à seis agentes comunitários da saúde (ACS), podendo ser inserido outros profissionais conforme a necessidade e condições do município. ${ }^{5}$ Neste processo de trabalho o enfermeiro exerce papel importante, ou seja, ele deve junto com sua equipe, planejar, implementar e avaliar as ações desenvolvidas pelo programa em sua área de abrangência.

Essa mudança de estratégia do sistema faz com que o enfermeiro adote a família como foco de cuidado, ${ }^{6}$ o que implica um desafio para sua prática profissional. ${ }^{7}$ Neste processo o enfermeiro deve conhecer cada família, identificando suas forças e fragilidades, suas reais necessidades e seus esforços, motivando-as a compartilhar responsabilidades.

Para atingir os objetivos propostos pelo PSF, ele deve atuar com autonomia e responsabilidade, procurando interagir com as famílias de forma eficaz e com resolutividade, atendendo suas necessidades.

Suas atribuições, estabelecidas pelo MS, vão desde a execução de ações de assistência básica de vigilância epidemiológica e sanitária nas áreas de atenção à criança, ao adolescente, à mulher, ao trabalhador e ao idoso, até a capacitação dos ACS e auxiliares de enfermagem, com vistas ao desempenho de suas funções junto ao serviço de saúde. O enfermeiro deve, também, oportunizar contatos com indivíduos sadios ou doentes, visando promover a saúde e abordar aspectos de educação em saúde, promovendo a qualidade de vida e 
contribuindo para que o meio ambiente torne-se mais saudável. Discutir, de forma permanente, junto à equipe de trabalho e comunidade, o conceito de cidadania, enfatizando os direitos à saúde e as bases legais que os legitimam, até a participação do processo de programação e planejamento das ações e da organização do processo de trabalho das Unidades Básicas de Saúde da Família (UBSF). ${ }^{4}$

Analisando essas atribuições e o contexto atual do PSF, percebemos quão importante é o papel do enfermeiro neste programa, pois ele é um dos responsáveis pelas famílias de sua área de abrangência, o que nos faz refletir sobre sua prática e desvendar suas ações.

Tentando conhecer as ações desenvolvidas pelos enfermeiros que atuam no PSF foi que realizamos este estudo cujo objetivo foi retratar a experiência vivida pelos enfermeiros que atuam em PSF, em uma Unidade Básica de Saúde de um município do Estado do Paraná.

\section{METODOLOGIA}

Trata-se de um estudo exploratório-descritivo realizado junto a 12 enfermeiros que atuam em PSF, em um município do Estado do Paraná, no período de maio à agosto de 2003. Uma pesquisa de natureza qualitativa tem como objetivo capturar o fenômeno estudado, ouvir e observar os sujeitos da pesquisa, e por fim dar a interpretação do que foi coletado. A opção por esse método de pesquisa deve-se ao fato dele possibilitar retratar e compreender a realidade do objeto em estudo. ${ }^{8}$ Para a obtenção dos dados utilizou-se uma entrevista semi-estruturada.

Os dados foram agrupados conforme semelhança de respostas, nos possibilitando construir categorias.

Este trabalho seguiu as normas da Resolução 196/96 do Ministério da Saúde, que regulamenta pesquisa com seres humanos. ${ }^{9}$

\section{ANÁLISE E DISCUSSÃO DOS DADOS}

Os dados obtidos foram discutidos, analisados e categorizados de forma a retratar o pensar e o agir dos enfermeiros que atuam em PSF, em um município do Estado do Paraná.

Eles nos possibilitaram construir as seguintes categorias: ATENDIMENTO DIFERENCIADO; SENTIMENTOS EXPERIENCIADOS; DIFICULDADES ENCONTRADAS, o que deu origem à categoria central: REALIDADE VIVIDA PELOS ENFERMEIROS QUE ATUAM EM PSF.

Em ATENDIMENTO DIFERENCIADO OS enfermeiros referem que o programa possibilita um atendimento específico a cada família e a seus membros, devido à possibilidade de acompanhar e visualizar a família como um todo, através do conhecimento de sua realidade.

“...A idéia de acompanhar a família como um todo é excelente, o profissional pode interagir com a família e direcionar melhor a conduta a ser tomada."

"Este programa visualiza a família como um todo, pressupondo que os problemas de saúde de um determinado membro familiar sempre estão ligados a problemas gerados no meio familiar."

"Uma vez que insere-se na realidade do paciente, o enfermeiro pode avaliar a condição familiar, o ambiente familiar levando em conta sua cultura, situação sócio-econômica, necessidades..."

Percebe-se nas falas a importância dada ao atendimento diferenciado para suprir as reais necessidades das famílias através da obtenção do conhecimento da realidade vivida por eles. Isto é obtido por meio da interação entre profissionais e famílias, preconizado pela UNICEF, quando refere que 
a Saúde da Família pode ser um dos instrumentos eficazes para prover bens e serviços, de modo diferenciado, para atender às distintas necessidades dos vários grupos populacionais, tendo em vista a redução das desigualdades. ${ }^{12}$

Da mesma forma, outros estudos reforçam que o atendimento diferenciado refere-se ao princípio, estabelecidos pelo programa, de igualdade, eqüidade e universalidade, pois a família deverá ser assistida conforme as suas reais necessidades. Para isso os profissionais que atuam em PSF devem interagir, conhecer e atender as necessidades específicas de cada família de sua área de atuação. 2,10,12

Em SENTIMENTOS EXPERIENCIADOS, os enfermeiros participantes deste estudo, relatam sentimentos positivos como sentir-se gratificado, satisfeito, realizado, contente e sentimentos negativos como desanimo, tristeza, frustração e impotência. Alguns relatam ambivalência de sentimentos. Isto pode ser observado nas falas a seguir:

"Muitas vezes gratificada, às vezes desanimada com sensação de impotência... na verdade, é contraditório, pois ao mesmo tempo que é satisfatório, também é triste, pois a sobrecarga de trabalho, o grande número de usuários e toda a burocracia envolvida dificulta o desenvolvimento adequado e uma atenção maior às famílias."

"Às vezes um pouco frustrada porque as comunidades não estão preparadas para esse tipo de atendimento, acho que precisaria um melhor esclarecimento. Enquanto profissionais temos muitas idéias para pôr em prática, mas não conseguimos a contento."

“O programa me realiza porque sou uma enfermeira mais assistencialista do que burocrática, faço muitos relatórios, supervisão técnica da equipe de Enfermagem, mas fico muito mais contente quando o usuário me procura e eu sei dos seus problemas, conheço sua casa, e ele tem confiança em mim."

Isso talvez se deva ao tipo de estratégias adotadas para desenvolver as ações do PSF, as quais são influenciadas pelas políticas locais, normas administrativas em vigor, herança cultural, preparo dos profissionais, hábitos, desconhecimento do programa pela comunidade e realidade encontrada. Estes fatores, muitas vezes, fazem com que o enfermeiro consiga atuar de forma diferenciada no seio das famílias, entretanto não consegue resolver todos os problemas, pois muitos dependem de outras instâncias para sua resolutividade. Os profissionais de saúde, atualmente, ainda agem sob a óptica biologicista, tecnicista e individualista, demonstrando dificuldades para lidar com outros tipos de atuação que envolvem necessidades e situações diferenciadas e específicas, fator este que pode gerar frustração. Isto pode ser agravado pelo fato de não haver recursos para o atendimento da demanda e pelo despreparo dos profissionais. ${ }^{13,14}$

Em DIFICULDADES ENCONTRADAS verificamos a existência de três sub-categorias: Falta de preparo para atuar em PSF, Tempo disponível e Necessidades de envolvimento.

Em "Falta de preparo para atuar em PSF" os sujeitos deste estudo referem que existe falta de reciclagem e de treinamento para que os profissionais possam desenvolver suas ações de forma eficiente e com resolutividade, proporcionando melhores condições e qualidade de vida às famílias.

“...falta de reciclagem dos profissionais para poderem executar as ações de enfermagem preconizadas pelo PSF..."

"Nós estávamos acostumados a desenvolver um tipo de trabalho, daí foi implantado o PSF, que preconiza outro tipo de ação e o treinamento que recebemos foi insuficiente." 
"Acho que houve falta de treinamento para atuarmos no programa, pois muita coisa mudou."

Verificamos que os enfermeiros sentem necessidade de maior preparo para desempenharem suas funções com maior segurança, eficiência e resolutividade. Eles desejam obter orientações de como proceder já que o sistema de saúde não mudou, mas as estratégias do PSF modificaram suas funções, pois antes o atendimento era realizado na USB, onde eram atendidas as necessidades de saúde do indivíduo, hoje, com o PSF, a assistência deve ser prestada à família exigindo dos profissionais conhecimento, interação, vínculo com as famílias e comunidades.

Atualmente a saúde coletiva vivência o paradigma da ambivalência do atendimento à saúde, pois de um lado temos o modelo hegemônico curativo e uma cultura consumista por parte da comunidade, e do outro lado a nova proposta de Atenção Básica a Saúde onde o foco é prevenção. ${ }^{14}$ É neste processo de trabalho que os enfermeiros sentem-se despreparados, e desatualizados, para atuarem de acordo com essa nova estratégia, onde eles devem agir, interagir e atender as necessidades das famílias e da comunidade, levando-os a serem co-responsáveis por esse processo, exercendo sua cidadania e obtendo melhor qualidade de saúde e de vida.

Em "Tempo disponível" os enfermeiros relataram falta de tempo para executar todas as ações preconizadas pelo PSF.

“...Tem pouco tempo para sair em área, fazer visitas, conhecer a realidade pois os funcionários são poucos e temos que fazer muitas coisas."

"Existe uma sobrecarga de atividades e funções, e não há tempo para fazer tudo o que se gostaria (...) isso dificulta o bom atendimento"
Observa-se que a falta de tempo é uma realidade vivida e expressa pelos enfermeiros do PSF, pois com a implementação deste programa houve sobrecarga de atividades e os recursos humanos continuaram deficientes. Isto retrata não apenas a falta de tempo para executar as ações de rotinas, mas, também, para implementar outras atividades que eles gostariam de realizar, para atender as especificidades e necessidades das famílias.

Isto pode gerar sentimento de "frustração", pois o profissional acredita que o seu trabalho não está sendo eficiente, que não está conseguido atender as reais necessidades sentidas pelas famílias e comunidade. ${ }^{13}$

Em "Necessidade de envolvimento" os sujeitos deste estudo referem como dificuldade para viabilizar o PSF a falta de envolvimento com o programa por parte de alguns profissionais. Eles atribuem este fato à herança cultural, valores, hábitos e preconceito.

“...alguns funcionários estão pouco envolvidos com o programa, pois não acreditam que ele mude as condições de saúde da comunidade..."

"Eles não estão acostumados a trabalhar em equipe (...) é difícil, antes do PSF cada um fazia sua parte..."

“...O não envolvimentos de alguns profissionais dificulta o trabalho em equipe. Eles não têm perfil para atuar no programa..."

"Para que o PSF funcione, como o Ministério quer, deve haver primeiro a quebra de alguns preconceitos entre os profissionais..."

Isto reflete o tipo de formação recebida pelos profissionais, que, em sua maioria, não foram preparados para atender as famílias de forma integral, 
As instituições formadoras, em geral, desenvolvem programas de ensino, cujo conteúdo programático esta, calcado em uma ciência individualista, biologicista, tecnicista e centrada na doença e no indivíduo.

Alguns profissionais não estão preparados e não apresentam perfil para desenvolver suas ações, de forma integrada, em equipe, na família e comunidade, entretanto fazem parte do PSF devido aos incentivos financeiros ofertados pelo programa.

O outro ponto, que deve ser enfocado, é a habilidade do profissional da saúde para trabalhar com o diferente, pois ele está acostumado a exercer uma postura paternalista, onde, muitas vezes, atua impedindo que as pessoas tomem decisões em relação ao seu bem-estar e saúde. No PSF as ações e decisões devem ser compartilhadas, portanto os profissionais de saúde devem rever seus valores, ideais, conhecimento, habilidade, preconceitos e postura profissional, para que atuem de forma eficaz e resoluta, permitindo que as famílias façam suas escolhas e tomem suas decisões conscientes em relação ao seu modo de ser e viver.

Neste processo, o papel do enfermeiro é assistir as famílias garantindo-lhes o acesso à informação, dando-Ihes o direito e a autonomia de decidir o que é melhor para sua saúde e qualidade de vida. ${ }^{11}$ Algumas atividades do PSF exigem que o enfermeiro trabalhe seus valores pessoais, seus conceitos de família e saúde para poder entender melhor as famílias por ele assistidas. ${ }^{13}$

Para isso ele deve procurar conhecer melhor o processo de ser e viver em família, refletindo sobre sua herança cultural, seus valores, hábitos, costumes, seus pontos fortes e fragilidades. Através da inserção harmônica, no seio das famílias, ele deve procurar conhecê-las e atender suas necessidades, propiciando-Ihes autonomia, sustentabilidade e melhor qualidade de vida.

\section{CONSIDERAÇÕES}

O PSF é uma tentativa de promover saúde e prevenir doenças e agravos, através de ações que envolvam as famílias no contexto onde estão inseridas, a comunidade. Todavia a forma como ele foi implantado e implementado, em algumas UBSF, faz com que suas ações não respondam as necessidades das famílias e o desejo expresso pelo MS.

Para a plena implementação do programa existem dificuldades, como a falta de pessoal em número e qualificação profissional adequada, falta de entrosamento entre profissionais, excesso de atividades gerando falta de tempo para desenvolvimento de ações, julgadas necessárias para se atingir a resolutividade da problemática encontrada, ou seja, para suprir as necessidades das famílias e das comunidades.

Os profissionais de Enfermagem que atuam em PSF estão satisfeitos com as estratégias estabelecidas pelo MS, pois elas possibilitam um atendimento diferenciado às famílias, através do seu acompanhamento na comunidade onde estão inseridos, pois isso possibilita maior interação e compreensão das realidades vividas pelas famílias, bem como, um melhor planejamento da assistência a ser realizada.

Ao desenvolverem as ações do PSF, enfermeiros vivenciam sentimentos como: realização, satisfação, contentamento, desânimo, tristeza, frustração e impotência. Esta ambivalência de sentimentos ocorre devido às dificuldades encontradas em seu processo de trabalho, as quais são ocasionadas pela falta de preparo profissional, tempo disponível para implementar as ações de Enfermagem, exigidas pelo programa, e necessidade de interação entre os membros da equipe.

Isso nos leva a concluir que: 1) Os enfermeiros que atuam em PSF desejam assistir/cuidar das famílias de forma humanitária, com eficiência e resolutividade, entretanto eles encontram dificuldades, umas ocasionadas pelo contraste de valores, hábitos e 
costumes transmitidos pela herança cultural; outras pela falta de preparo e conhecimento para interagir junto às famílias e os demais membros da equipe; e pela sobrecarga de trabalho resultante da escassez de recursos humanos. 2) Para que o PSF seja executado de acordo com o preconizado pelo MS, os enfermeiros e demais componentes da equipe devem ser melhor preparados pelos órgãos formadores, para exercerem suas atividades de forma harmônica, eficaz e com resolutividade, proporcionando às famílias melhores condições de vida e o exercício da cidadania. As instituições de ensino, portanto,devem formar profissionais aptos a desenvolverem as estratégias do programa, ou seja, não só para atuarem no processo saúde/doença. 3) As instituições gerenciadoras das políticas de saúde, quer a nível municipal, estadual ou federal, devem suprir as UBSF com recursos humanos qualificados e recursos financeiros necessários para a execução das ações estabelecidas no referido programa. Eles devem, também, disponibilizar apoio específico para que os profissionais possam enfrentar sentimentos e conflitos produzidos pelo processo de trabalho desenvolvido em PSF, pois esses sentimentos podem ser desgastante e atingir a saúde dos profissionais, em especial a do enfermeiro. Neste processo, ele deve ser preparado para situações de conflito e instrumentalizados para saber como trabalhar com as questões sociais que emergem da comunidade onde atuam. 4) Os órgãos gestores das políticas de saúde e as instituições de ensino devem oportunizar educação continuada para estas equipes e, também, o desenvolvimento de pesquisas para poder suprir necessidades e apontar soluções.

Acreditamos que o PSF seja capaz de assistir/cuidar da saúde das famílias de forma diferenciada, mas para que isso se concretize com eficiência e resolutividade os gestores das políticas de saúde devem priorizar não só o atendimento das necessidades sentidas pela população, mas, também, devem investir na capacitação e bem estar dos profissionais que atuam no programa, oferecendo recursos adequados para o desenvolvimento de suas ações.

Ao atuar em PSF, os enfermeiros devem estar conscientes de que sua ação junto às famílias não só oportunizará melhores condições e qualidade de vida de seus membros, mas, também, transformará a realidade vivida, através do exercício consciente e pleno de sua cidadania.

Por outro lado, eles irão adquirir maior credibilidade e reconhecimento junto à comunidade, o que fará com que aumente sua auto-estima e poder de ação.

\section{REFERÊNCIAS}

1 Marques RS, Mendes A. Atenção Básica e Programa de Saúde da Família (PSF): novos rumos para a política de saúde e seu financiamento? Ciência \& Saúde Coletiva 2003; 8(2); 403-15.

2 Senna MCM. Equidade e política de saúde: algumas reflexões sobre o Programa Saúde da Família. Caderno de Saúde Pública, Rio de Janeiro, 2002; 18(supl.); 203-211.

3 Ministério da Saúde (BR). Lei Orgânica da Saúde, $\mathrm{n}^{\circ}$ 8.080, 19 de setembro 1990. Conselho Nacional de Secretários Municipais de Saúde. Publicações Técnicas. no 2, dez. 1990.

4 Ministério da Saúde (BR). Uma estratégia para reorientação do modelo assistencial. Brasília, 1997.

5 Ministério da Saúde (BR). Programa de Saúde da Família. Disponível em: < http://www.saúde.gov.br/psf/ programa/index.asp > (05 jun. 2003).

6 Motta MGC, Luz AMH. Família como unidade de desenvolvimento humano e saúde. Ciência, Cuidado e Saúde 2003; 2(supl.); 24-7.

7 Santos BRL et al. Formando o enfermeiro para o cuidado à saúde da família: um olhar sobre o ensino de graduação. Revista Brasileira de Enfermagem 2000; 53(esp.); 49-59.

8 Minayo MCS. O desafio do conhecimento: pesquisa qualitativa em saúde. Rio de Janeiro: Hucitec; 1994. 
9 Ministério da Saúde (BR). Conselho Nacional de Saúde. Resolução no 196 de 10 de Out. 1996. Diretrizes e Normas Regulamentadoras de Pesquisa de envolvendo Seres Humanos. Brasília; 1996.

10 Sousa MF. A Enfermagem reconstruindo sua prática: mais que uma conquista no PSF. Revista Brasileira de Enfermagem 2000; 53(esp.); 25-30.

11 Fortes PAC, Martins CL. A ética, a humanização e a saúde da família. Revista Brasileira de Enfermagem 2000; 53(esp.); 31-3.
12 Bousso RS, Angelo M. A Enfermagem e o cuidado na saúde da família. Manual de Enfermagem, p. 18-22, 2001. Disponível em: < http:/wwwids-saude.org.br/ enfermagem > (12 jun. 2003).

13 Nascimento E. Programa Saúde da Família: vivenciando a experiência. Revista Brasileira de Enfermagem 2000; 53(esp.); 171-3.

14 Pedrosa JIS, Teles JBM. Consenso e diferenças em equipes do Programa Saúde da Família. Revista de Saúde Pública 2001; 35(3); 303-11. 